\title{
Rapid bone loss occurs as early as 2 days after complete spinal cord transection in young adult rats
}

\author{
Yuanzhen Peng ${ }^{1} \cdot$ Wei Zhao ${ }^{1} \cdot$ Yizhong $\mathrm{Hu}^{2} \cdot \mathrm{Fei} \mathrm{Li}^{3} \cdot$ X. Edward Guo ${ }^{2}$. Dong Wang ${ }^{4}$ William A. Bauman ${ }^{1,5,6}$. \\ Weiping Qin ${ }^{1,5}$
}

Received: 13 May 2019 / Revised: 19 September 2019 / Accepted: 20 September 2019 / Published online: 29 October 2019

(c) The Author(s), under exclusive licence to International Spinal Cord Society 2019

\begin{abstract}
Study design Animal study.

Objective This study examined how soon after spinal cord injury (SCI) bone loss occurs, and investigated the underlying molecular mechanism.

Methods Eight-week-old male Wistar rats underwent complete transection of the thoracic spinal cord at T3-4 or sham operation ( $n=10-12$ per group). Blood, hindlimb bone samples, and bone marrows were collected at 2 and 7 days after SCI. Results The neurologically motor-complete SCI causes loss of bone mass and deterioration of trabecular bone microstructure as early as 2 days after injury; these skeletal defects become more evident at 7 days. These changes are associated with a dramatic increase in levels of bone resorption maker CTX in blood. Alternations of gene expression in hindlimb bone tissues and bone marrow cells at the first week after SCI were examined. Gene expressions responsible for both bone resorption and formation are increased at 2 days post-SCI, and the associated bone loss and bone deterioration are likely the result of higher levels of osteoclastic resorption over osteoblastic formation, as may be extrapolated from findings at molecular levels.

Conclusions Rapid bone loss occurs as early as 2 days after motor-complete SCI and interventions for inhibiting bone resorption and prompting bone formation should start as soon as possible after the injury to prevent bone loss.
\end{abstract}

These authors contributed equally: Yuanzhen Peng, Wei Zhao

Supplementary information The online version of this article (https:// doi.org/10.1038/s41393-019-0371-4) contains supplementary material, which is available to authorized users.

Weiping Qin

weiping.qin@mssm.edu

1 National Center for the Medical Consequences of Spinal Cord Injury, James J. Peters VA Medical Center, Bronx, NY, USA

2 Department of Biomedical Engineering, Columbia University, New York, NY, USA

3 Yantaishan Hospital, Yantai, Shandong, China

4 Department of Pharmaceutical Sciences, College of Pharmacy, University of Nebraska Medical Center, Omaha, NE, USA

5 Departments of Medicine, Icahn School of Medicine at Mount Sinai, New York, NY, USA

6 Rehabilitation Medicine, Icahn School of Medicine at Mount Sinai, New York, NY, USA

\section{Introduction}

Spinal cord injury (SCI) is a devastating neurological disorder that causes extreme immobilization with immediate and irreversible unloading of the skeleton and leads to marked bone loss. In individuals with neurologically motorcomplete SCI, bone mass may be diminished by more than $50 \%$ at the distal femur and proximal tibia [1-4]. Consequently, fractures of the distal femur and proximal tibia are the most common fractures in individuals with SCI [5, 6]. In humans with SCI, BMD declines rapidly at all sublesional skeletal sites at rates as high as $1 \%$ per week for the first year after injury $[1,4,7]$. In studies performed in acute models of SCI in rats, loss of trabecular bone in the proximal tibia approaches $70 \%$ at 3 weeks after a complete spinal cord transection [8] and over $60 \%$ at the distal femoral metaphysis at 10 days after severe spinal cord contusion [9]. Recent studies reported that SCI reduced bone mass and caused deterioration of trabecular bone microstructure as early as 1 week after injury [10-12]. Considering the fact that as much as $-50 \%$ of reduction in 
bone mass can be induced by SCI within a short period time of 1 week [12], we hypothesize that bone loss can occur even much earlier time points after SCI.

To date, the mechanisms underlying bone loss due to SCI remain largely unknown. As such, at the present time no practical treatment has been reported to delay or prevent bone loss, or promote rebuilding of bone, in patients with SCI [13]. A better understanding of the molecular mechanism and risk factors for SCI-related bone loss is critical in identifying therapeutic targets to preserve the mass and structural integrity of bone and reduce fracture and associated co-morbidity. Uncoupled bone turnover after SCI has been reported, including a reduced bone formation rate, as well as marked acceleration of bone resorption associated with increased osteoclast surface and increased potential of bone marrow progenitors for osteoclastic differentiation [1, 2, 7, 14]. In contrast, coupled bone remolding has been also observed. In addition to dramatically increased bone resorption, the osteoblast number and activity are unaffected or even elevated at 3 weeks after SCI in rats [8] and, similarly, bone formation markers are at normal or slightly above normal levels in SCI patients at 3 months post injury [15, 16].

Bone resorption is stimulated by cells of the osteoblast lineage by the release of receptor activator of NF-kB ligand (RANKL), which stimulates differentiation and activity of osteoclasts [17]. RANKL is probably responsible for much of the bone loss resulting from various states of immobilization, such as that occurring after SCI [17]. Cells of the osteoblast lineage release osteoprotegerin (OPG), a decoy receptor which inhibits RANKL [17]. Osteocytes, which are derived from osteoblasts after they become encased in bone, are the major source of RANKL in unloaded bone [18]. The $\mathrm{Wnt} / \beta$-catenin pathway is a key determinant of bone mass by controlling osteoblast function, and that regulation of this pathway in bone by Wnt inhibitors contributes to the regulation of bone mass [17, 19]. Wnt inhibitors linked to bone metabolism include sclerostin, Dickkopf-1 (DKK1) and frizzled-related proteins (sFRPs) and play important roles in the pathogenesis of disuse osteopenia [20]. Sclerostin and DKK1 act by binding to the Wnt co-receptor LRP5/6 thereby inhibiting Wnt signaling, whereas sFRPs bind Wnts to reduce their biological activity [20]. Sclerostin is encoded by the SOST gene to potently inhibits bone formation and is produced by bone cells, particularly osteocytes [21]. Recent clinical studies have demonstrated that serum sclerostin levels are elevated after acute SCI [22]. Most recently, administration of sclerostin antibody prevented or reversed bone loss by increasing bone formation and restoring deterioration of osteocyte morphology/ structure in rat models of acute SCI [23-25], suggesting that sclerostin probably contributes to the modulation of SCIinduced bone loss.
The purpose of this study was to examine how rapidly bone loss can occur after SCI in young adult rats and to investigate underlying molecular mechanism. A rat model of complete spinal cord transection at the midthoracic level was used for these studies as we previously described [24, 26-28]. Because the majority of persons with $\mathrm{SCI}$ are male, male rats were selected for this work. The authors report herein that SCI induces bone loss and structural deterioration as early as 2 days after injury, at least in part, as a consequence of an excess of osteoclastic resorption over that of osteoblastic formation, as evidenced and extrapolated from molecular findings. The findings from the present study have implication in bone loss after acute SCI, particularly in clinical cases of children or Veterans with SCI. The latter is of particular interest since Veterans Health Administration has the largest single network of SCI care in the nation [29].

\section{Methods}

\section{Animals surgery, drug administration, and tissue collection}

Animals were maintained on a 12:12-h light/dark cycle with lights on at $07: 00 \mathrm{~h}$ in a temperature-controlled $(20 \pm$ $2{ }^{\circ} \mathrm{C}$ ) vivarium, and all procedures were approved by the James J. Peters Veterans Affairs JJP VA Medical Center Institutional Animal Care and Use Committee. Spinal cord transection surgery was performed as previously described [24-28]. In brief, 9-week-old male Wistar rats (Charles River) were anesthetized by inhalation of isofluorane (3-5\%) and hair were removed with a clipper. Skin over the back was cleaned with betadine and isopropyl alcohol. After making a midline incision, the spinal cord at the site of transection (T3-4) was visualized by laminectomy, and the spinal cord was transected with microscissors. The space between transected ends of the spinal cord was filled with surgical sponge and the wound was sutured closed in two layers. Urine was manually expressed three times daily until bladder automaticity developed, then this procedure was performed as needed. Baytril was administered for the first 3-5 days postoperatively then as needed for cloudy or bloody urine as a sign of probable urinary tract infection or for evidence of wound infection. Sham-transected animals received only a laminectomy.

Body weights were recorded prior to spinal cord transection (preoperative body weight) and daily after SCI. Body weight at sacrifice was normalized relative to preoperative body weight. Two or seven days after SCI, animals were euthanized by inhalation of isofluorane prior to harvesting the tissue for study. The leg was removed using sterile technique; 
careful dissection was performed to free the head of the femur from the pelvis. To preserve bone for micro-CT ( $n=6-7$ per group), the leg was removed and placed into tubes containing 4\% PFA overnight, after which the PFA was replaced with $70 \%$ ethanol for storage. The femur and tibia $(n=5-6$ per group) were placed in ice-cold Minimum Essential Alpha Medium ( $\alpha$-MEM), and then immediately processed for extraction of total RNA from whole bone and bone marrow cell cultures, as described below.

\section{Dual-energy X-ray absorptiometry}

Areal BMD measurements were performed on excised hindlimbs ( $n=10-12$ animals per group) by using a small animal dual-energy X-ray absorptiometer (DXA) (Lunar Piximus, WI, USA) as described [24-28, 30, 31]. Hindlimbs were positioned on the DXA platform with the knee flexed at an angle of $135^{\circ}$ and DXA images were acquired with Lunar Pixmus software. The instrument was calibrated with a phantom following the procedures recommended by the manufacturer on each day of use prior to analysis of experimental samples. The metaphysis of the distal femur and proximal tibia were selected as regions of interest (ROI). The coefficient of variation for the repeated measurements for the ROI was $\sim 1.5 \%$.

\section{Micro-CT analysis of bone microarchitecture}

Bone architecture of the distal femur was assessed by a Scanco $\mu C T$ scanner (vivaCT 80; Scanco Medical AG, Switzerland) at $21 \mathrm{~mm}$ isotropic voxel size, as previously described [24-27, 32] (also see the Supplemental Materials and Methods for greater details). Image reconstruction and 3D quantitative analysis were performed using software provided by Scanco. Scans were initiated at the distal end of the femur and extended to the center of the femur, for a total of $\sim 777$ slices $(\sim 16.3 \mathrm{~mm})$. Trabecular ROI consisted of 189 slices $(\sim 3.969 \mathrm{~mm})$, beginning $0.5 \mathrm{~mm}$ proximal to the growth plate and continuing in a proximal direction, were included in the bone analysis. Cortical ROI consisted of 100 slices at the proximal end of the scans, located $\sim 2.1 \mathrm{~mm}$ at the center of the femur, were also analyzed. Standard nomenclature and methods for bone morphometric analysis were employed [24, 32].

Mechanical properties at the distal femur trabecular bones were estimated from micro-finite element analysis ( $\mu \mathrm{FEA})$, following the manufacturer's recommended procedures, as previously described $[2,25,33-35]$. Briefly, $\mu$ FEA models were produced by converting each bone voxel to an eightnode brick element. Bone tissue was subjected to applied uniaxial compression, with an elastic modulus of $15 \mathrm{GPa}$ and Poisson's ratio of 0.3 for each element. A linear elastic analysis was used to estimate the bone stiffness.

\section{Serum levels of CTX and osteocalcin}

Blood samples ( $n=10-12$ animals per group) were clotted at room temperature for $15 \mathrm{~min}$ and centrifuged at $2000 \mathrm{rpm}$ for 10 min at $4{ }^{\circ} \mathrm{C}$. After that, the serum was separated and then stored at $-80^{\circ} \mathrm{C}$. Serum C-terminal telopeptide of type I collagen (CTX) levels were measured using a RatLapsTM enzyme-immunoassay kit from Immunodiagnostic Systems (Fountain Hills, AZ). Serum concentrations of osteocalcin were measured using a rat osteocalcin enzyme-linked immunoassay kit from Biomedical Technologies Inc. (Stoughton, MA). All samples were assayed in duplicate, following the manufacturer's recommended procedures.

\section{Extraction of total RNA from bone}

Total bone RNA was extracted as previously described with some modifications [36]. Briefly, long bones were dissected free of soft tissues, and bone marrow were flushed away with PBS using a $27 \mathrm{G}^{1 / 2}$ needle-syringe. The bone samples $(\sim 1 \mathrm{~g})$ were longitudinally cut into small piece and then digested three times with $2 \mathrm{mg} / \mathrm{ml}$ collagenase type I (Gibco, >150 Units; $20 \mathrm{ml}$ ), one time with $5 \mathrm{mM}$ EDTA (Sigma-Aldrich, $10 \mathrm{ml})$ ), and one more with the collagenase and EDTA, each for $25 \mathrm{~min}$ on a shaker with rotation at $150 \mathrm{rpm}$ at $37^{\circ} \mathrm{C}$. Following the digestions, the bone samples were crushed using a mortar and pestle in liquid nitrogen. RNA was extracted from the lysate using the TRizol reagent (Sigma Aldrich) according to the manufacturer's instructions.

\section{Extraction of total RNA from bone marrow cultures}

Procedures for the differentiation of osteoblasts and osteoclasts from bone marrow stem cells were performed, as previously described [25-28, 30]. Briefly, to study osteoclast cells, bone marrow cells were isolated from the femora and tibiae in $\alpha$-MEM. Marrow cells were rinsed, resuspended in $\alpha$-MEM, seeded into wells using an equal number of cells in each well, and then cultured for 2 days in $\alpha$-MEM supplemented with human macrophage colony-stimulating factor (M-CSF; $5 \mathrm{ng} / \mathrm{ml}$ ). The nonadherent cells were collected and purified by Ficoll-Plus (Amersham Pharmacia Biotech Inc., Arlington Height, IL, USA) then seeded into wells, again with an equal number of cells per plate, and incubated in $\alpha$-MEM containing M-CSF $(30 \mathrm{ng} / \mathrm{ml})$ and RANKL $(60 \mathrm{ng} / \mathrm{ml})$ for 5 days, followed by total RNA extraction using the TRizol reagent. To study osteoblast cells, cells were flushed from the marrow cavity with $\alpha$-MEM and seeded into tissue culture wells; the harvested bone marrow cells were cultured in $\alpha$-MEM supplemented with $15 \%$ preselected FCS (Hyclone, Logan, UT, USA) and ascorbic acid-2-phosphate $(1 \mathrm{mM})$. Recruitment of marrow stromal cells to the osteoblast lineage was assessed at 


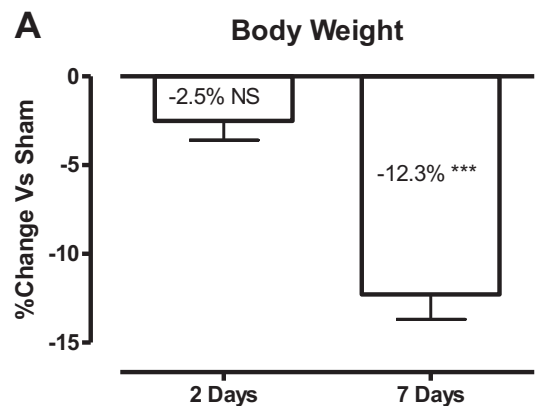

B

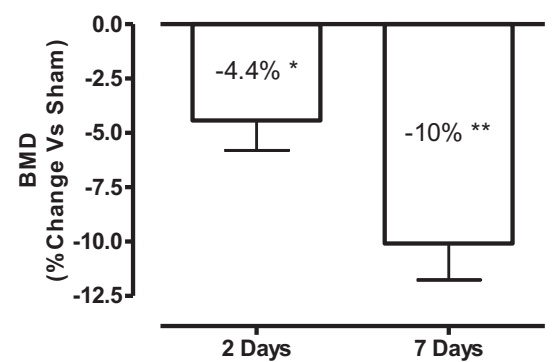

C

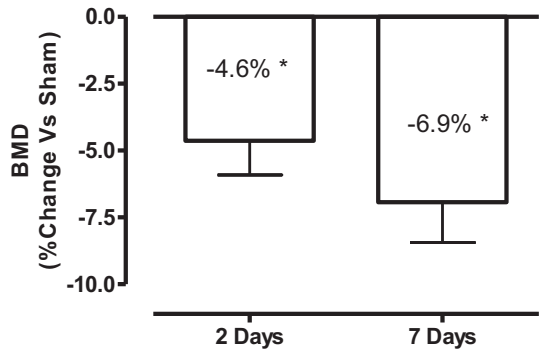

Fig. 1 Rapid loss of bone mass after acute SCI. a Change in total body weight at 2 and 7 days after SCI is shown. Body weight at sacrifice was normalized relative to body weight prior to spinal cord transection (preoperative body weight). Areal bone mineral density (BMD) measurements in each group are shown at the distal femur (b) and proximal tibia (c). The percent change versus Sham group was calculated at

10 days of culture by extraction of total RNA with the TRizol reagent.

\section{Quantitative PCR}

Real-time PCR was used for the determination of mRNA levels, as described previously [24, 28, 30]. One microgram of total RNA was used to synthesize the first strand cDNA by the High Capacity cDNA Reverse Transcription Kit (Applied Biosystems). qPCR was performed with an Applied Biosystems (ABI) Via 7 thermal cycler using ABI Taqman 2X PCR mix and ABI Assay on demand qPCR primers. Changes in expression were calculated using the $2^{-\Delta \Delta \mathrm{Ct}}$ method using $18 \mathrm{~S}$ RNA as the internal control $[24,28,30]$.

\section{Statistics}

Data are expressed as mean $\pm \mathrm{SD}$; the number of independent samples (n) is provided in the legend of each figure. The statistical significance of differences among means was tested using Student's $t$-test to determine the significance of differences between individual pairs of means using a $\mathrm{p}$ value of 0.05 as the cutoff for significance. Statistical calculations were performed using Prism 4.0c (GraphPad Software, La Jolla, CA, USA).

\section{Results}

\section{Rapid loss of bone mass and deterioration of bone microstructure after acute $\mathrm{SCl}$}

Average total body weight started to decline at 2 days after SCI and were significantly lower in the SCI-vehicle group at 7 days compared with the Sham-SCI group (Fig. 1a).

Compared with Sham rats, BMD was significantly lower in SCI groups both at the distal femur $(-4.4 \%, p<0.05$;
2- and 7-days post-SCI for each outcome measurement and then was plotted in the graph. Data are expressed as mean \pm SEM. $n=10-12$ animals per group. Significance of differences was determined using Student's $t$ test. $* P<0.05$, $* * P<0.01$, and $* * * p<0.001$ versus the indicated group; NS no significant difference

Fig. 1b) and at the proximal tibia $(-4.6 \%, p<0.05$; Fig. 1c) as early as 2 days after injury. At 7 days post injury, BMD was further lower at the distal femur $(-10 \%, p<0.01$; Fig. 1b) and at the proximal tibia $(-6.9 \%, p<0.05$; Fig. 1c) when compared with Sham animals.

Bone architecture was examined by high-resolution $\mu \mathrm{CT}$ to assess the changes in trabecular bone of the distal femur (Fig. 2 and Supplementary Table 1). At two days post injury, lower trabecular bone volume (BV/TV, $-18.5 \%$, $p<0.05$; Fig. 2b, panel a), trabecular bone number (Tb.N, $-13.7 \%, p<0.05$; Fig. 2b, panel b), trabecular bone thickness (Tb.Th, $-8.7 \%$, no significance; Fig. 2b, panel c), connectivity density (Conn-Dens, $-20.6 \%$, no significance; Fig. $2 b$, panel e), and stiffness $(-39.2 \%$, no significance; Fig. $2 b$, panel g) with an increased trabecular separation $(+5.4 \%$, no significance; Fig. $2 \mathrm{~b}$, panel d) and a higher value for the structure model index $(+26.4 \%$, no significance; Fig. 2b, panel f) were observed in SCI groups when compared with Sham animals. At 7 days after SCI, the deterioration of trabecular bone microstructure had progressed further with a significant reduction of BV/TV $(-28.8 \%, p<0.01$; Fig. $2 \mathrm{~b}$, panel a), trabecular bone number $(-15.8 \%, p<0.05$; Fig. $2 \mathrm{~b}$, panel b), trabecular bone thickness $(-11.4 \%, p<0.05$; Fig. $2 b$, panel c), connectivity density $(-43.7 \%, p<0.05$; Fig. $2 \mathrm{~b}$, panel e), and stiffness $(-52.8 \%, p<0.05$; Fig. $2 \mathrm{~b}$, panel g) with a significant increase in trabecular separation $(+24.3 \%, p<0.01$; Fig. 2b, panel d) and a larger value for the structure model index $(+36.2 \%$, no significance; Fig. $2 b$, panel f).

Cortical bone structure at the femur midshaft was also examined by high-resolution $\mu C T$ (Supplemental Fig. 1a). Compared with those from the Sham group, bones at 7 days after SCI tended to be thinner with a reduction in Ct.Ar/Tt.Ar $(-7.0 \%, p<0.05$; Supplemental Fig. 1b, panel a) and Ct.Th $(-7.1 \%, p<0.05$; Supplemental Fig. 1b, panel b) and an increase in medullary area $(+15.6 \%, p<0.05$; Supplemental Fig. 1b, panel c). These 
A

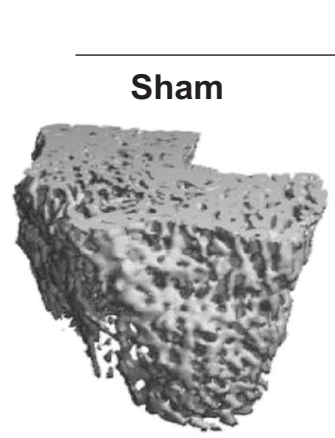

2 days

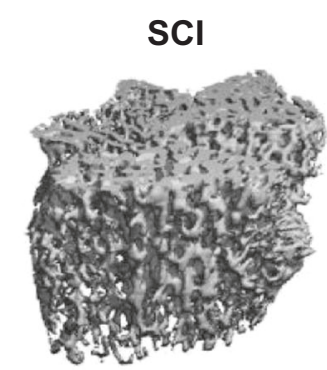

7 days

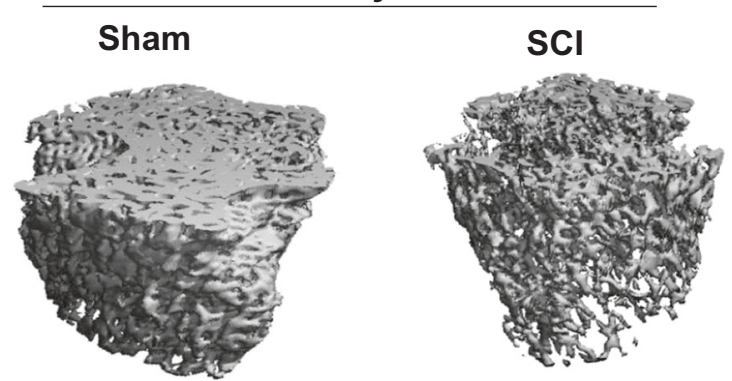

B (a)

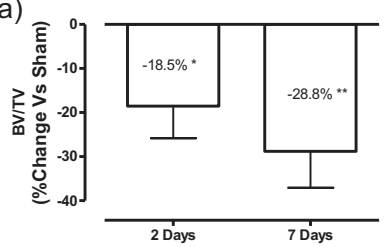

(e)

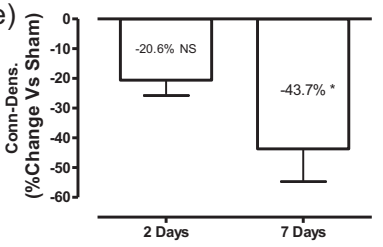

(b)

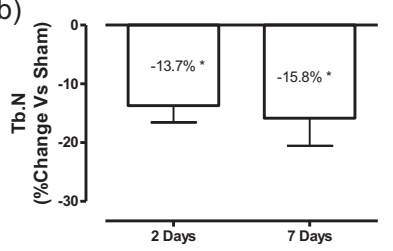

(f)

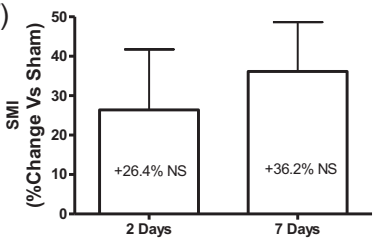

(c)

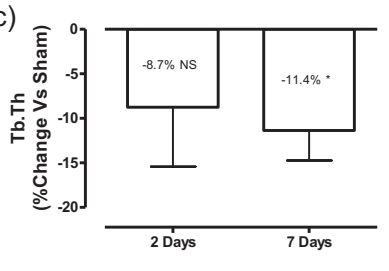

(g)

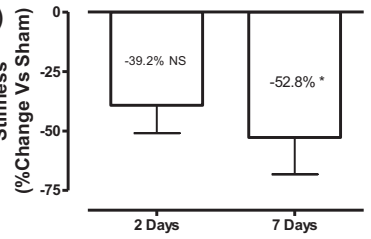

(d)

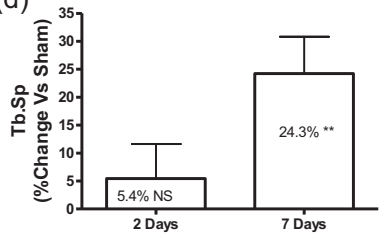

Fig. 2 Rapid deterioration of bone microstructure after acute SCI. a Representative micro-CT 3D images of trabecular microarchitecture of distal femur are displayed. b Measurements are shown for: (panel a) trabecular bone volume per total tissue volume (BV/TV), (panel b) trabecular number (Tb.N, mm ${ }^{-1}$ ), (panel c) trabecular thickness $(\mathrm{Tb}$. Th $(\mathrm{mm})$ ), (panel d) trabecular separation (Tb.Sp (mm)), (panel e) connectivity density (conn.D $\left(\mathrm{mm}^{-3}\right)$ ), and (panel f) structure model index (SMI; range from 0 to 3 , with $0=$ platelike and $3=$ rod like). (panel g) Bone stiffness was estimated from micro-finite element analysis $(\mu \mathrm{FEA})$. The percent change versus Sham group was calculated at 2- and 7-days post-SCI for each outcome measurement and then was plotted in the graph. Data are expressed as mean \pm SEM. $n=$ 6-7 animals per group. Significance of differences was determined by using Student's $t$-test. $* P<0.05$ and $* * P<0.01$ versus the indicated group. NS not significant structural changes of the diaphysis were not observed at 2 days after SCI.

\section{Changes of circulating bone markers and bone gene expressions at 2 days after $\mathbf{S C l}$}

To understand molecular mechanisms underlying bone loss after acute SCI, serum levels bone metabolic products for reflecting bone resorption (CTX) and bone formation (osteocalcin) were examined. We found that when compared with Sham rats, protein levels of serum CTX and osteocalcin in SCI groups were higher by about 2 - and 1.5 -fold in animals at 2 days after injury, respectively (Fig. 3a). In addition, total RNAs from whole bone of hindlimb were extracted and gene expressions responsible for bone resorption and formation were analyzed by quantitative PCR analysis. At 2 days post injury, increases of the osteoclastic markers RANKL $(p<$ 0.001 , Fig. 3b, panel a), TRAP ( $p<0.05$, Fig. 3b, panel b), Intergrin ( $p<0.05$, Fig. 3b, panel c), and calcitonin receptor (CTR) $(p<0.01$, Fig. 3b, panel d) were detected in bone samples from SCI group when compared with Shamtransected animals. Interestingly, increases of the osteoblastic markers Runx2 $(p<0.001$, Fig. 3c, panel a) and osteocalcin $(p<0.01$, Fig. $3 \mathrm{c}$, panel b) as well as SOST mRNA $(p<0.001$, Fig. 3c, panel d) were also observed in SCI group. There are no significant changes in mRNA levels of DKK1 (Fig. 3c, panel e), DKK2 (data not shown), and sFRP1 (Fig. 3c, panel f).

\section{Changes of circulating bone markers and bone gene expressions at 7 days after $\mathrm{SCl}$}

Serum CTX levels were higher by about 1.5 -fold in SCI groups at 2 days after injury when compared with Sham rats. Serum osteocalcin levels had no significant change at 7 days after SCI (Fig. 4a). In ex vivo cultures of osteoclasts derived from bone marrow hematopoietic precursors, significant increase of the osteoclastic markers TRAP $(p<$ 0.05 , Fig. $4 \mathrm{~b}$, panel a) was observed in SCI group at 7 days post injury when compared with Sham-transected animals, 


\section{A. Serum bone markers}
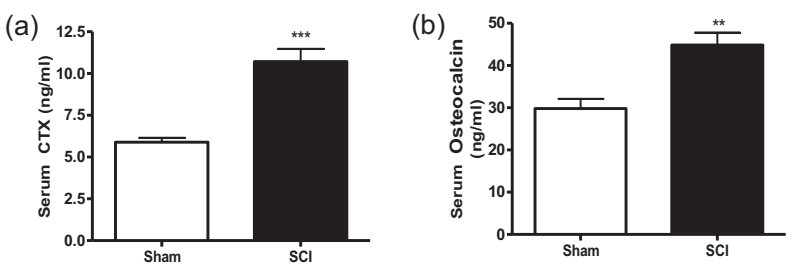

B. Bone resorption related genes (a)

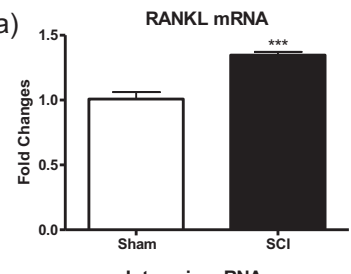

(c)

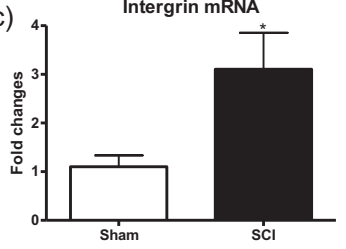

(b)

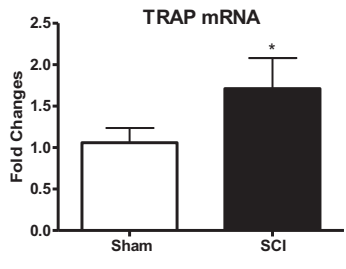

(d)

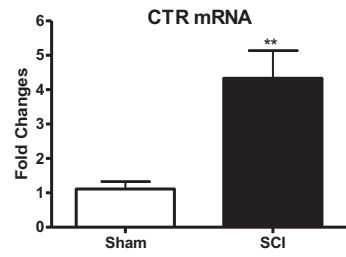

Fig. 3 Changes in circulating bone markers and bone gene expression at 2 days after SCI. a Serum CTX (panel a) and osteocalcin (panel b) levels by ELISA. Using RNA extracted from long bones, gene expressions related to bone resorption (b) and bone formation and Wnt signaling pathway (c) were determined by real-time PCR analysis: (b, panel a) RANKL, (b, panel b) TRAP, (b, panel c) intergrin $\beta 3$, (b, panel d) Calr, (c, panel a) Runx2, (c, panel b) osteocalcin, (c, panel c)

but no significant change was found for RANKL (Fig. 4b, panel b), CTR (Fig. 4b, panel c), and Intergrin (Fig. 4b, panel c). In ex vivo cultures of osteoblasts derived from bone marrow stromal precursors, although mRNA levels for the differentiation marker Runx2 (Fig. 4c, panel a), osteocalcin (Fig. 4c, panel b), and BSP (Fig. 4c, panel c) did not significantly change compared with Sham group (Fig. 4c, panel a), marked increases of mRNA levels were observed for the Wnt signaling inhibitors SOST $(+30 \%, p<0.05$; Fig. 4c, panel d), DKK1 $(+1,043 \%, p<0.001$; Fig. 4 c, panel e), DKK2 $(+190 \%, p<0.05$; Fig. $4 c$, panel f), and sFRP1 $(+212 \%, p<0.05$; Fig. $4 c$, panel g) in the SCI group compared with the Sham group.

\section{Discussion}

Bone loss after $\mathrm{SCI}$ is relatively unique in its magnitude, rapidity, and localization to the sublesional regions. We previously showed that substantial loss of bone mineral content and bone volume was found for the distal femur and proximal tibia of rats at 56 days after spinal cord transection $[24,25,27,28]$. Recent studies demonstrated that SCI causes bone loss and deterioration of trabecular bone microstructure at 10 days [9] or even as early as 1 week

\section{Bone formation and Wnt signaling-related genes}

(a)

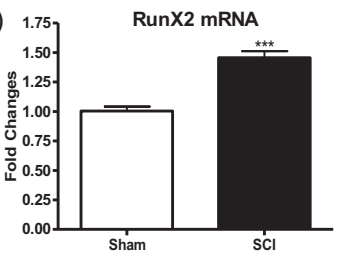

(c)

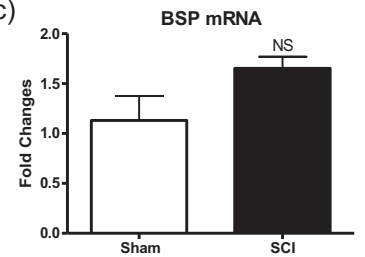

(e)

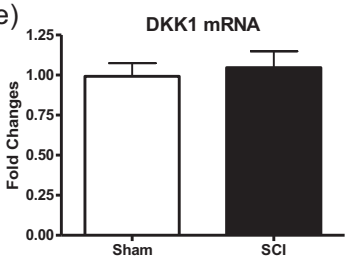

(b) $2.57 \quad$ Osteoclacin mRNA

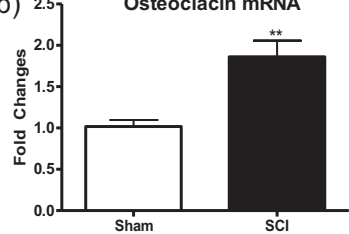

(d) SOST mRNA

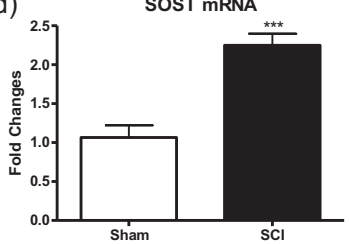

(f)

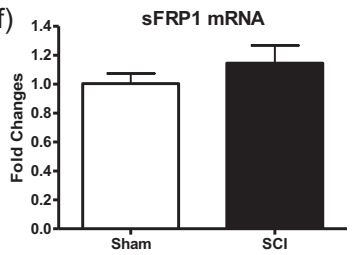

OPG, (c, panel d) SOST, (c, panel e) DKK1, and (c, panel f) sFRP1. Gene expression was normalized to $18 \mathrm{~s}$ RNA. Data are expressed as mean \pm SEM. $n=10-12$ animals per group for (a) and $n=5-6$ animals per group for (b) and (c). Significance of differences was determined by using Student's $t$-test. $* p<0.05$, ** $p<0.01$, and $* * * p$ $<0.001$ versus the indicated group. NS no significant difference

[10-12] after injury. The present study is the first to report that bone loss and deterioration of trabecular bone microstructure occur as early as 2 days after neurologically motor-complete SCI. Specifically, SCI results in to rapid reduction of sublesional cancellous bone mass [e.g., BMD and bone volume (BV/TV)] as well as deleterious microarchitectural changes (e.g., increased Tb.Sp and higher SMI for rod-like geometry, as well as reduced Tb.N, Conn.D and stiffness), indicative of compromised structural integrity and reduced mechanical strength. These skeletal deficits due to SCI become more evident at 7 days, which is consistent with the previous findings [10-12]. The rapid decline in bone mass and deterioration of bone microstructure highlight a clinical need that any intervention for the prevention of bone loss after the acute event should start as soon as possible after paralysis and immobilization.

Our data indicate that sublesional loss of BMD within the first 2 days of SCI in rodents is as much as $4.4-4.6 \%$, a degree of bone loss that has been observed after 1 year of menopause in women who are not taking osteo-protective drugs [37]. The reasons for such a rapid decline in bone mass and deterioration of bone microstructure after SCI remain unclear. Bone mass and integrity are maintained by a balance between bone resorption and formation that is mediated by osteoclasts and osteoblasts, respectively [17]. The process is 
A. Serum bone markers

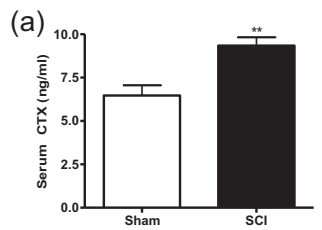

(b)

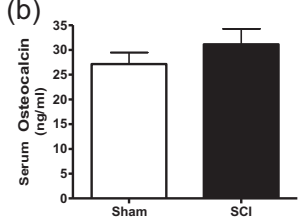

B. Bone resorption related genes

(a)

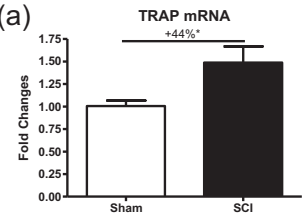

(c)

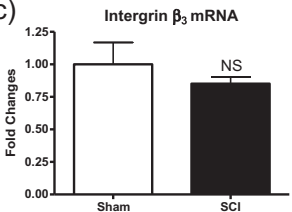

(b)

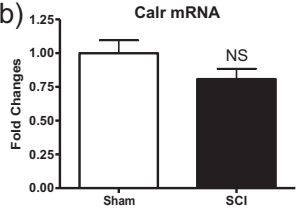

(d)

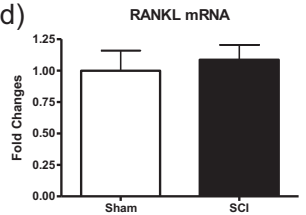

C. Bone formation and Wnt signaling-related genes
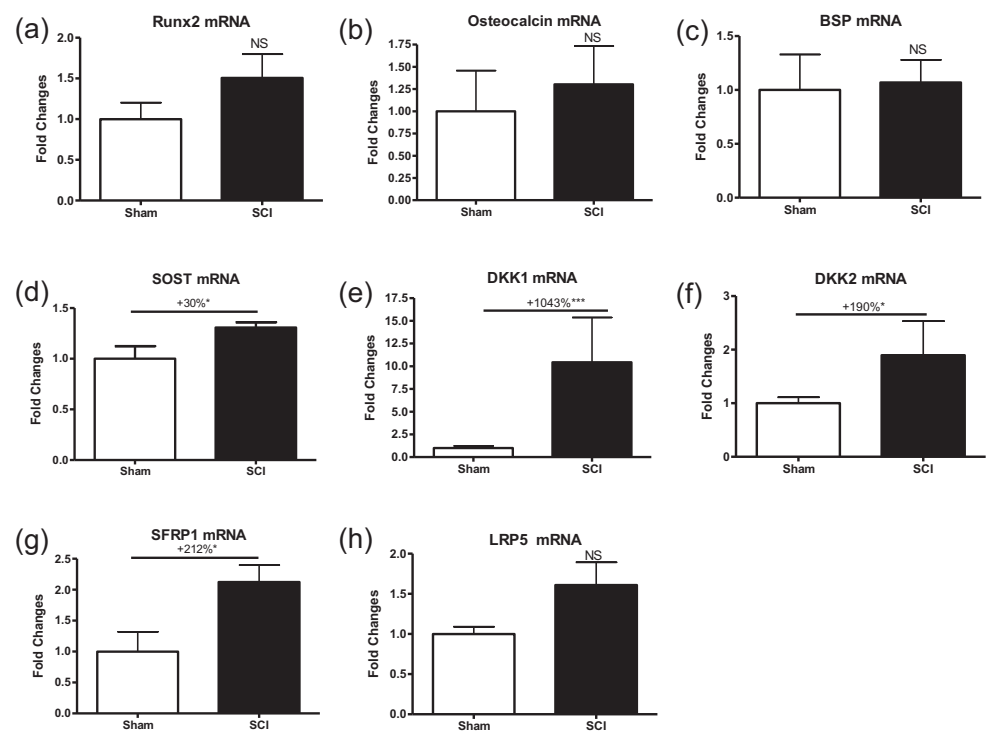

Fig. 4 Changes in circulating bone markers and bone gene expression at 7 days after SCI. a Serum CTX (panel a) and osteocalcin (panel b) levels by ELISA. b, c mRNA levels were determined by real-time PCR. b Gene expression in osteoclasts developed by primary culture of bone marrow hematopoietic cells. (b, panel a) TRAP, (b, panel b) Calr, (b, panel c) Intergrin $\beta 3$, (b, panel d) RANKL. c Gene expression in osteoblasts developed by primary culture of bone marrow stromal cells. (c, panel a) Runx2, (c, panel b) osteocalcin, (c, panel c) BSP, (c, panel d) SOST, (c, panel e) DKK1, (c, panel f) DKK2, (c, panel g) sFRP1, and (c, panel h) LRP5. Gene expression was normalized by 18s RNA. Data are expressed as mean \pm SEM. $n=10-12$ animals per group for (a) and $n=5-6$ animals per group for (b) and (c). Significance of differences was determined by using Student's $t$-test. $* P<0.05$ and $* * * p<0.001$ versus the indicated group. NS no significant difference regulated by multiple factors in a coordinated fashion, including local and systemic hormones, mechanical loading and neuromuscular activity $[4,17,31]$. An abnormality of any one of these factors may rapidly lead to bone loss. For example, estrogen deficiency contributes to bone loss in women after menopause; [17] weightlessness and immobility, as experienced by astronauts or bedridden or otherwise immobilized patients, leads to reduced bone mass and strength; several neurologic diseases, such as stroke, amyolotropic lateral sclerosis, Parkinson's disease and polio cause loss of bone due to abnormal neuromuscular function [4, 38]. In contrast to each of the aforementioned pathological conditions that cause bone loss, SCI results in neurological dysfunction (denervation), extreme immobilization, and alterations of several hormones. As such, the multifaceted pathophysiological alterations in patients after acute SCI contribute to one of the rapidest and most severe forms of bone loss that will eventually result in a high risk for fragility fracture in those with chronic SCI [4, 31, 39].

The present study, for the first time, demonstrates evidence of alternations in gene expression responsible for bone resorption and formation in hindlimb bone tissues and bone marrow stem cells at 1 week after SCI. The molecular mechanisms underlying SCI-induced bone loss remain largely unknown [13]. Currently, there is inconsistent evidence as to whether coupling or uncoupling of bone resorption and formation occurs subsequent to SCI. For example, uncoupled bone turnover after SCI has been reported, including a reduced bone formation rate, as well as marked acceleration of bone resorption associated with increased osteoclast surface and increased potential of bone marrow progenitors for osteoclastic differentiation [1, 7, 14]. On the other hand, the coupled bone remolding has been also observed. In addition to dramatically increased bone resorption, the osteoblast number and activity are unaffected or even elevated at 3 weeks after SCI in rats [8] and, similarly, bone formation markers are at normal or slightly above normal levels in SCI patients at 3 months post injury $[15,16]$.

One of the main findings is that bone starts an accelerated process of remodeling immediately after injury, probably mediated, among other factors, by a sudden increase in SOST expression. In this study, we found the increased expression of three osteoclast markers in hindlimb bone tissues collected from SCI rats at 2 days after injury, suggesting rapid activation of osteoclast cells. We also observed marked increases in expression of RANKL, a key factor which stimulates osteoclast formation from marrow progenitor cells [17], consistent with increased RANKL expression by osteocytes in other models of bone unloading [18]. Increases of the osteoblastic markers Runx2 and osteocalcin were also observed in SCI group. The findings of increased SOST mRNA (suggesting reduced bone formation) and simultaneously increased RunX2 and osteocalcin (suggesting increased bone formation) at 2 days after 
SCI are of great interest. The observation is consistent with increases in CTX and osteocalcin immediately after ovariectomy $[40,41]$. The evidence that increased SOST, CTX, and osteocalcin expression occur simultaneously at this time point suggests an increase in bone remodeling, thereby resulting in immediate increase in the activities of both osteoclast and osteoblasts. Because osteoclastic resorption is more rapid (2-3 days) than osteoblastic bone deposition (2-3 weeks), initially resorption predominates with a net loss in bone mass. In addition, the increased mRNA levels of RunX2 and osteocalcin are perhaps associated with a compensatory mechanism to increase bone formation in response to excessive bone resorption activity at this time point. Importantly, mRNA levels for RANKL is significantly higher at 2 days, but did not significantly change at 7 days after SCI, compared with Sham animals. Collectively, these findings indicate that gene expressions essential for bone resorption and formation are both increased at the earliest stages after acute SCI, suggesting that the associated bone loss and bone deterioration are likely attributed from an excess of RANKL-mediated osteoclastic resorption over that of osteoblastic formation, as may be extrapolated from molecular findings.

Interestingly, levels of SOST mRNA expression were increased both at 2 and 7 days after SCI, further supporting the roles of sclerostin in modulating SCI-related bone loss, as previous studies have suggested [22-25]. However, at 7 days after SCI, there is a different pattern with regard to changes of other gene expressions. Although significant increase of the osteoclastic marker TRAP was noted, mRNA levels for the RANKL, a key osteoclastic differentiation factor, did not significantly change compared with Sham animals, supporting the notion that at this time point, bone resorption is still activated with a diminished degree. Similarly, osteoblast differentiation marker Runx2, osteocalcin and BSP did not significantly change compared with Sham animals.

In addition to SOST, expressions of other Wnt signaling inhibitors DKK1, DKK2, and sFRP1 mRNAs were markedly increased, suggesting that bone formation is likely suppressed very early after SCI and perhaps this explains the relative ineffectiveness of antiresorptive medications in completely preventing bone loss after SCI in previous studies $[1,13,38]$. The findings also imply that SOST acts in a swift manner to directly respond to mechanical unloading signals while the response from other Wnt pathway molecules DKK1, DKK2, and sFRP1 is somewhat delayed. Collectively, the findings from this study indicate that bone resorption and formationmediated remodeling and bone adaptation after acute SCI is a result of dynamic molecular events guiding a shift of bone remodeling from coupling at 2 days post injury to uncoupling at 7 days, with resulting net catabolism of the sublesional skeleton. This notion is further supported by the evidence of bone metabolic products in blood circulation. At 2 days after
SCI, both bone resorption marker serum CTX and formation maker serum osteocalcin are elevated, but the magnitude of change in serum CTX ( $\sim 2$ fold) is more robust than that of serum osteocalcin $(\sim 1.5$-fold; Fig. 3a), suggesting that there is a coupled bone remodeling with a predominance of resorption over formation. A similar predominant resorption is also observed at 7 days after SCI where levels of serum CTX remains increased by $\sim 1.5$-fold, in absence of a coordinated change in serum osteocalcin (Fig. 4a).

In summary, the present study has demonstrated for the first time that the neurologically motor-complete SCI causes loss of bone mass and deterioration of trabecular bone microstructure as early as 2 days after injury. Our findings suggest that SCIinduced bone loss and structural deterioration during early stages of injury are likely resulting from an excess of osteoclastic resorption over osteoblastic formation at molecular levels. Thus, interventions for inhibiting bone resorption and prompting bone formation should start as soon as possible after the injury to prevent the early onset of bone loss.

\section{Data availability}

The datasets generated and/or analysed during the current study are available from the corresponding author on reasonable request.

Funding This work was supported by the Veterans Health Administration, Rehabilitation Research and Development Service (Grants 5I01RX001313 and 5I01RX02089-A2 to WQ; B9212-C and B2020-C to $\mathrm{WAB})$.

Author contributions WAB and WQ were responsible for study design and data analysis. YP, WZ, FL, YH, DW, and EG performed the experimental procedures to generate the data for the study. This paper was written by WQ and was revised and approved by all authors. WQ takes responsibility for the integrity of the data analysis.

\section{Compliance with ethical standards}

Conflict of interest The authors declare that they have no conflict of interest.

Ethics statement We certify that all applicable institutional and governmental regulations concerning the ethical use of animals were followed during the course of this research.

Publisher's note Springer Nature remains neutral with regard to jurisdictional claims in published maps and institutional affiliations.

\section{References}

1. Qin W, Bauman WA, Cardozo C. Bone and muscle loss after spinal cord injury: organ interactions. Ann N Y Acad Sci. 2010; 1211:66-84.

2. Lin T, Tong W, Chandra A, Hsu SY, Jia H, Zhu J, et al. A comprehensive study of long-term skeletal changes after spinal cord injury in adult rats. Bone Res 2015;3:15028. 
3. Bauman WA, Cardozo C. Immobilization Osteoporosis. In: Marcus R, Nelson D, Rosen CJ, editors. Osteoporosis Fourth Edition, Cambridge, Massachusetts: Academic Press; 2013.

4. Qin W, Bauman WA, Cardozo CP. Evolving concepts in neurogenic osteoporosis. Curr Osteoporos Rep. 2010;8:212-8.

5. Akhigbe T, Chin AS, Svircev JN, Hoenig H, Burns SP, Weaver FM, et al. A retrospective review of lower extremity fracture care in patients with spinal cord injury. J Spinal Cord Med. 2015;38:2-9.

6. Morse LR, Battaglino RA, Stolzmann KL, Hallett LD, Waddimba A, Gagnon D, et al. Osteoporotic fractures and hospitalization risk in chronic spinal cord injury. Osteoporos Int 2009;20:385-92.

7. Dudley-Javoroski S, Shields RK. Muscle and bone plasticity after spinal cord injury: review of adaptations to disuse and to electrical muscle stimulation. J Rehabil Res Dev. 2008;45:283-96.

8. Jiang SD, Jiang LS, Dai LY. Changes in bone mass, bone structure, bone biomechanical properties, and bone metabolism after spinal cord injury: a 6-month longitudinal study in growing rats. Calcif Tissue Int. 2007;80:167-75.

9. Morse L, Teng YD, Pham L, Newton K, Yu D, Liao WL, et al. Spinal cord injury causes rapid osteoclastic resorption and growth plate abnormalities in growing rats (SCI-induced bone loss in growing rats). Osteoporos Int 2008;19:645-52.

10. Yarrow JF, Ye F, Balaez A, Mantione JM, Otzel DM, Chen C, et al. Bone loss in a new rodent model combining spinal cord injury and cast immobilization. J Musculoskelet Neuronal Interact. 2014;14:255-66.

11. Del Rivero T, Bethea JR. The effects of spinal cord injury on bone loss and dysregulation of the calcium/parathyroid hormone loop in mice. Osteoporos Sarcopenia. 2016;2:164-9.

12. Minematsu A, Nishii Y, Imagita H, Sakata S. Time course of changes in trabecular bone microstructure in rats with spinal cord injury. J Life Sci. 2014;8(No. 6):522-8.

13. Battaglino RA, Lazzari AA, Garshick E, Morse LR. Spinal cord injury-induced osteoporosis: pathogenesis and emerging therapies. Curr Osteoporos Rep. 2012;10:278-85.

14. Otzel DM, Conover CF, Ye F, Phillips EG, Bassett T, Wnek RD, et al. Longitudinal examination of bone loss in male rats after moderate-severe contusion spinal cord injury. Calcif Tissue Int. 2019;104:79-91.

15. Maimoun L, Couret I, Micallef JP, Peruchon E, Mariano-Goulart D, Rossi M, et al. Use of bone biochemical markers with dualenergy x-ray absorptiometry for early determination of bone loss in persons with spinal cord injury. Metabolism 2002;51:958-63.

16. Roberts D, Lee W, Cuneo RC, Wittmann J, Ward G, Flatman R, et al. Longitudinal study of bone turnover after acute spinal cord injury. J Clin Endocrinol Metab. 1998;83:415-22.

17. Zaidi M. Skeletal remodeling in health and disease. Nat Med 2007;13:791-801.

18. Xiong J, Onal M, Jilka RL, Weinstein RS, Manolagas SC, O'Brien CA. Matrix-embedded cells control osteoclast formation. Nat Med 2011;17:1235-41.

19. Glass DA 2nd, Bialek P, Ahn JD, Starbuck M, Patel MS, Clevers $\mathrm{H}$, et al. Canonical Wnt signaling in differentiated osteoblasts controls osteoclast differentiation. Dev Cell 2005;8:751-64.

20. Bonewald LF, Johnson ML. Osteocytes, mechanosensing and Wnt signaling. Bone 2008;42:606-15.

21. Bonewald LF. The amazing osteocyte. J Bone Min Res. 2011;26:229-38.

22. Battaglino RA, Sudhakar S, Lazzari AA, Garshick E, Zafonte R, Morse LR. Circulating sclerostin is elevated in short-term and reduced in long-term SCI. Bone 2012;51:600-5.

23. Beggs LA, Ye F, Ghosh P, Beck DT, Conover CF, Balaez A, et al. Sclerostin inhibition prevents spinal cord injury-induced cancellous bone loss. J Bone Min Res. 2015;30:681-9.
24. Qin W, Li X, Peng Y, Harlow LM, Ren Y, Wu Y, et al. Sclerostin antibody preserves the morphology and structure of osteocytes and blocks the severe skeletal deterioration after motor-complete spinal cord injury in rats. J Bone Min Res. 2015;30:1994-2004.

25. Zhao W, Li X, Peng Y, Qin Y, Pan J, Li J, et al. Sclerostin antibody reverses the severe sublesional bone loss in rats after chronic spinal cord injury. Calcif Tissue Int. 2018;103:443-54.

26. Qin W, Sun L, Cao J, Peng Y, Collier L, Wu Y, et al. The Central Nervous System (CNS)-independent anti-bone-resorptive activity of muscle contraction and the underlying molecular and cellular signatures. J Biol Chem. 2013;288:13511-21.

27. Qin W, Zhao W, Li X, Peng Y, Harlow LM, Li J, et al. Mice with sclerostin gene deletion are resistant to the severe sublesional bone loss induced by spinal cord injury. Osteoporos Int. 2016;27:3627-36.

28. Sun L, Pan J, Peng Y, Wu Y, Li J, Liu X, et al. Anabolic steroids reduce spinal cord injury-related bone loss in rats associated with increased Wnt signaling. J Spinal Cord Med. 2013;36:616-22.

29. U.S. Department of Veterans Affairs VA: Expands Spinal Cord Injury Care. 2000. https://www.va.gov/opa/pressrel/pressrelease. $\mathrm{cfm}$ ?id $=204$.

30. Bramlett HM, Dietrich WD, Marcillo A, Mawhinney LJ, FuronesAlonso O, Bregy A, et al. Effects of low intensity vibration on bone and muscle in rats with spinal cord injury. Osteoporos Int 2014;25:2209-19.

31. Cardozo CP, Qin W, Peng Y, Liu X, Wu Y, Pan J, et al. Nandrolone slows hindlimb bone loss in a rat model of bone loss due to denervation. Ann N Y Acad Sci 2010;1192:303-6.

32. Bouxsein ML, Boyd SK, Christiansen BA, Guldberg RE, Jepsen KJ, Muller R. Guidelines for assessment of bone microstructure in rodents using micro-computed tomography. J Bone Min Res. 2010;25:1468-86.

33. Pistoia W, van Rietbergen B, Lochmuller EM, Lill CA, Eckstein F, Ruegsegger P. Estimation of distal radius failure load with micro-finite element analysis models based on three-dimensional peripheral quantitative computed tomography images. Bone 2002;30:842-8.

34. Lan S, Luo S, Huh BK, Chandra A, Altman AR, Qin L, et al. 3D image registration is critical to ensure accurate detection of longitudinal changes in trabecular bone density, microstructure, and stiffness measurements in rat tibiae by in vivo microcomputed tomography (muCT). Bone 2013;56:83-90.

35. Liu XS, Zhang XH, Sekhon KK, Adams MF, McMahon DJ, Bilezikian JP, et al. High-resolution peripheral quantitative computed tomography can assess microstructural and mechanical properties of human distal tibial bone. $\mathrm{J}$ Bone Min Res. 2010;25:746-56.

36. Kitase Y, Vallejo JA, Gutheil W, Vemula H, Jahn K, Yi J, et al. beta-aminoisobutyric Acid, 1-BAIBA, Is a Muscle-Derived Osteocyte Survival Factor. Cell Rep 2018;22:1531-44.

37. Berarducci A. Stopping the silent progression of osteoporosis. Am Nurse Today. 2009;3:18.

38. Bauman WA, Cardozo CP. Osteoporosis in individuals with spinal cord injury. PM R 2015;7:188-201.

39. Jiang SD, Jiang LS, Dai LY. Mechanisms of osteoporosis in spinal cord injury. Clin Endocrinol. 2006;65:555-65.

40. Yoon KH, Cho DC, Yu SH, Kim KT, Jeon Y, Sung JK. The change of bone metabolism in ovariectomized rats: analyses of microCT scan and biochemical markers of bone turnover. J Korean Neurosurg Soc. 2012;51:323-7.

41. Szulc P, Delmas PD. Biochemical markers of bone turnover: potential use in the investigation and management of postmenopausal osteoporosis. Osteoporos Int 2008;19:1683-704. 